\title{
Accuracy of surface integral equation matrix elements in plasmonic calculations
}

\author{
T. V. Raziman, ${ }^{1}$ W. R. C. Somerville, ${ }^{2}$ O. J. F. Martin, ${ }^{1, *}$ and E. C. Le $\mathbf{R u}^{2,3}$ \\ ${ }^{1}$ Nanophotonics and Metrology Laboratory, Swiss Federal Institute of Technology Lausanne (EPFL), \\ 1015 Lausanne, Switzerland \\ ${ }^{2}$ The MacDiarmid Institute for Advanced Materials and Nanotechnology, School of Chemical and Physical Sciences, \\ Victoria University of Wellington, P.O. Box 600, Wellington 6140, New Zealand \\ ${ }^{3}$ e-mail: eric.leru@vuw.ac.nz \\ ${ }^{*}$ Corresponding author: olivier.martin@epfl.ch
}

Received November 17, 2014; revised January 7, 2015; accepted January 24, 2015; posted January 29, 2015 (Doc. ID 227019); published February 27, 2015

\begin{abstract}
We study the convergence of the integrals required to be evaluated in the surface integral equation (SIE) formulation (or method of moments) for simulating the optical response of plasmonic nanostructures. We analyze how the numerical quadratures used to compute the integrals affect the accuracy of the SIE matrix elements and, in turn, that of the relevant physical quantities calculated using the method. Based on these studies, we propose an optimized algorithm for evaluation of the integrals, which improves the accuracy of the results without significantly increasing the calculation overhead. @ 2015 Optical Society of America

OCIS codes: (250.5403) Plasmonics; (050.1755) Computational electromagnetic methods; (290.5825) Scat-

tering theory.

http://dx.doi.org/10.1364/JOSAB.32.000485
\end{abstract}

\section{INTRODUCTION}

Electromagnetic fields at optical frequencies can excite propagating waves confined to metal-dielectric interfaces (surface plasmon polaritons) as well as nonpropagating electromagnetic modes in metallic nanostructures (localized surface plasmons) [1]. Both kinds of excitations have been studied extensively, and the field of plasmonics has grown considerably in the recent past. Plasmonics has found applications in diverse fields such as biological and chemical sensing [2], design of devices such as solar cells [3] and photodetectors [4], and enhancing optical effects as in surfaceenhanced spectroscopy [5] or nonlinear optics []. ]. Designing plasmonic structures with desired optical properties has become a field of active pursuit, and the ability to accurately simulate the optical response of these structures is of paramount importance.

Various numerical methods are used to simulate the optical response of plasmonic nanostructures in the time and frequency domains. The most commonly used ones include finite difference time-domain [7,8], finite element method [9], discrete dipole approximation [10], volume integral equation [11], the $T$-matrix method $[12,13]$, boundary element method (BEM) [14,15], and surface integral equation (SIE) [16, 17], also called method of moments (MoM) outside the plasmonics community [18]. The SIE approach solves Maxwell's equations in the frequency domain in the integral form and provides many advantages over the other methods. The technique requires the discretization of only the boundaries of scatterers reducing computational overhead, in terms of both time and memory, and does not need the artificial truncation of space as might be required by methods that discretize the background as well $[19,20]$. SIE requires the computation of
Green's tensor, which is straightforward for homogeneous domains but not for general media. SIE formulations have been recently developed to deal with periodic structures [21] and stratified backgrounds [22] by computing Green's tensor for these cases.

Even though the surface integral equations themselves are completely accurate within the realm of classical electrodynamics, any numerical implementation would only be able to solve them approximately. The various fields and currents are expanded in terms of a finite number of basis functions, which can only approximate the actual values. This problem can be addressed by using higher-order basis functions or finer meshes, but it increases computational costs tremendously in $3 \mathrm{D}$ problems [23]. In addition, the $4 \mathrm{D}$ integrals required in the calculation of the matrix elements involving the Green tensor have to be done numerically. If the scatterer surface is discretized into $N$ elements, $O\left(N^{2}\right)$ such integrals will have to be computed, and using complicated algorithms to calculate the integrals accurately can be prohibitively expensive. It is therefore desirable to use low-order integration routines, as long as it does not compromise the accuracy, and even validity, of the results. These integration problems are magnified by the singular nature of the integrand for some integrals involving overlapping or nearby elements. In the general context of the MoM, much work has been dedicated to improving the integral accuracy using schemes of singularity subtraction [24-26] or improved numerical integration $[27,28]$.

In this paper, we focus specifically on the relevance of these problems for the modeling of optical properties of metallic nanostructures. We study the link between numerical quadrature order and integral accuracy and how it relates 
to the accuracy of the physical quantities calculated by SIE. Since we are mostly interested in finely meshed subwavelength structures in plasmonics, wave effects due to the change of phase of Green's tensor over a triangular element are not of primary concern. However, the singular behavior of Green's tensor at the source causes a large variation in amplitude over triangular elements close to it. This latter effect is numerically more relevant in plasmonics. Hence, we will be addressing specifically the geometric singularities rather than wave effects.

\section{SURFACE INTEGRAL EQUATIONS FOR PLASMONIC NANOSTRUCTURES}

\section{A. SIE Implementation for Plasmonics}

There exist various formulations to solve surface integral equations numerically, with varying numerical accuracy $[29,30]$. In this paper, we will be following the formulation in [16]. Here, we provide a brief description of this SIE implementation, but only the most relevant formulas are given, as full details can be found elsewhere [16].

Surface currents are expanded in terms of the standard Rao-Wilton-Glisson basis functions [31], which are also used as test functions (Galerkin's method) to obtain the equations in matrix form. The tangential boundary values of the surface integral equations are used, and the Poggio-MillerChang-Harrington-Wu-Tsai formulation [32-34] is chosen to combine the integral equations over domains. For two domains, i.e., a metallic object embedded in a dielectric medium, the SIE matrix can then be written as (here, we use the same notations as in [16])

$$
\left[\begin{array}{cc}
\mathbf{D}^{1}+\mathbf{D}^{2} & -\left(\mathbf{K}^{1}+\mathbf{K}^{2}\right) \\
\left(\mathbf{K}^{1}+\mathbf{K}^{2}\right) & \frac{1}{Z_{1}^{2}} \mathbf{D}^{1}+\frac{1}{Z_{2}^{2}} \mathbf{D}^{2}
\end{array}\right] .
$$

With the $n$th basis functions denoted $\mathbf{f}_{n}(\mathbf{r})$, the matrix elements can be expressed as reaction integrals in terms of the scalar Green's functions in each medium, i.e., $G_{i}\left(\mathbf{r}, \mathbf{r}^{\prime}\right)=$ $\exp \left(i k_{i}\left|\mathbf{r}-\mathbf{r}^{\prime}\right|\right) /\left(4 \pi\left|\mathbf{r}-\mathbf{r}^{\prime}\right|\right)$ :

$$
\begin{aligned}
D_{n k}^{i}= & \frac{i Z_{i}}{k_{i}} \int_{S_{n}} \mathrm{~d} S \nabla \cdot \mathbf{f}_{n}(\mathbf{r}) \int_{S_{k}} \mathrm{~d} S^{\prime} G_{i}\left(\mathbf{r}, \mathbf{r}^{\prime}\right) \nabla^{\prime} \cdot \mathbf{f}_{k}\left(\mathbf{r}^{\prime}\right) \\
& -i k_{i} Z_{i} \int_{S_{n}} \mathrm{~d} S \mathbf{f}_{n}(\mathbf{r}) \cdot \int_{S_{k}} \mathrm{~d} S^{\prime} G_{i}\left(\mathbf{r}, \mathbf{r}^{\prime}\right) \mathbf{f}_{k}\left(\mathbf{r}^{\prime}\right), \\
K_{n k}^{i} & =\int_{S_{n}} \mathrm{~d} S \mathbf{f}_{n}(\mathbf{r}) \int_{S_{k}} \mathrm{~d} S^{\prime}\left[\nabla^{\prime} G_{i}\left(\mathbf{r}, \mathbf{r}^{\prime}\right)\right] \times \mathbf{f}_{k}\left(\mathbf{r}^{\prime}\right) .
\end{aligned}
$$

Note that the matrices $\mathbf{D}$ and $\mathbf{K}$ are symmetric, as can be deduced directly from the expressions above or more formally as a consequence of optical reciprocity.

The surfaces $S_{n}$ and $S_{k}$ are each formed from two triangles with a common edge (indexed by $n$ and $k$ ); the above integrals are therefore computed by summing the integrals over each of the four possible triangle pairs. Note that these integrals are $4 \mathrm{D}$ and can be carried out as two successive $2 \mathrm{D}$ integration on the source $\left(\mathbf{r}^{\prime}\right)$ and test (r) triangles.

Two types of 2D numerical quadratures for the triangle will be considered to study the effect of the number of integration points on accuracy:
- Symmetrical Gaussian triangular quadratures, as derived in [35], which will be called Dunavant quadrature for short. The quadrature orders used here are $n_{O}=1,2,3,4,5,6,7$, $10,13,17$, and 19, which correspond to $N_{q}=1,3,4,6,7$, $12,13,25,37,61$, and 73 integration points, respectively.

- Uniform subdivision of the triangle, consisting of subdividing each edge into $n$ elements, resulting in dividing the area into $n^{2}$ congruent subtriangles. The centers of the subtriangles are the $N_{q}=n^{2}$ quadrature points. The quadrature orders used are 21, 30, and 40, corresponding to $N_{q}=441,900$, and 1600 integration points, respectively.

Note that, for a given $N_{q}$, the total number of quadrature points for the $4 \mathrm{D}$ integral is $N_{q}^{2}$.

In practice, the singular nature of Green's function at $\mathbf{r}=\mathbf{r}^{\prime}$ can be problematic for the numerical evaluation of those integrals when the triangles are close to each other or overlapping. This problem can be mitigated using various schemes of singularity subtraction (SS). We here follow the SS method described in [16]. Source integrals over $\mathbf{r}^{\prime}$ are evaluated by separating Green's function into a smooth part and a singular part, explicitly with $R=\left|\mathbf{r}-\mathbf{r}^{\prime}\right|$ :

$$
G^{i}(R)=G_{\text {sing }}^{i}(R)+G_{\text {smooth }}^{i}(R),
$$

with

$$
G_{\text {sing }}^{i}(R)=\frac{1}{4 \pi}\left(\frac{1}{R}-\frac{k_{i}^{2} R}{2}\right)
$$

The first term is trivially singular at $R=0$, while the second results in a singularity for $\nabla G^{i}$. The singular part can be integrated analytically over $\mathbf{r}$ [26], while the smooth part is integrated numerically without accuracy issues. In both cases, the test integral (over $\mathbf{r}^{\prime}$ ) must still be integrated numerically. Because this scheme breaks the symmetry between source and test integrals, the symmetry of the matrices is no longer guaranteed by construction and can therefore be used to validate the accuracy of the computed matrices.

The formulation presented above has been used in the context of plasmonics with $N_{q}=1$ (and is then similar to the BEM $[\underline{14}, \underline{15}])$ and shown to produce promising results for metallic nanostructures in the near- and far-field zones [16]. Here, we will study its accuracy in detail.

\section{B. Additional Improvements}

In the course of this study, a couple of additional techniques were also added to improve the accuracy, as will be shown in the next section.

First, within the SS scheme, the double integrals involving the dominant term of $G_{\text {sing }}^{i}(R)$ (i.e., $1 /(4 \pi R)$ ) can be computed entirely analytically for identical triangles in $\mathbf{D}$, using the formulas of [36]. The remaining integral involving $G^{i}(R)-$ $1 /(4 \pi R)$ is then integrated numerically without any singularity issues.

Second, for nonidentical triangles, it has been pointed out in [25] that there also remains a "small" logarithmic singularity for $\overline{\mathbf{K}}$ associated with the second term in $G_{\text {sing }}^{i}(R)$, which may cause problems in the numerical integration over the test triangle. Reference [25] also proposed to overcome this problem by changing the order of integration and replacing the outer integral (now over the source triangle) by a line integral over 
its edges \{Eq. (22) in [25]\}. This approach was implemented and will be referred as the SSL method (singularity subtraction with line integral). In these cases, the line integral is computed using a standard Gauss-Legendre quadrature with $N_{l}=4 n_{O}$ quadrature points, chosen to be of the same order as for the 2D Dunavant quadratures (i.e., for $n_{O}=17$, we have $N_{l}=68$ and $N_{q}=61$ ).

\section{Computing the Optical Properties}

The SIE solution is obtained by inversion of the SIE matrix (using direct inversion with the mldivide algorithm in MATLAB), and the surface currents on each triangular mesh element are then derived. From these, the fields at any point can, in principle, be computed from a surface integral [16]. This integral can, however, be problematic for points close to the surface. Moreover, the computation of the scattering cross section by integration of the fields over a surface far from the particle is also inefficient. To avoid those problems, the optical properties here were obtained directly from the electric and magnetic surface currents as follows. The surface currents are defined as [16] ( $\mathbf{n}$ is the normal pointing outward)

$$
\mathbf{J}=\mathbf{n} \times \mathbf{H}_{\text {surf }} \quad \text { and } \quad \mathbf{M}=-\mathbf{n} \times \mathbf{E}_{\text {surf }} .
$$

From those and Maxwell's equations, we deduce the surface fields at any point just outside the particle (domain 1) as

$$
\begin{gathered}
\mathbf{E}_{\text {surf }}=\mathbf{n} \times \mathbf{M}-\frac{i Z_{1}}{k_{1}}(\nabla \cdot \mathbf{J}) \mathbf{n}, \\
\mathbf{H}_{\text {surf }}=-\mathbf{n} \times \mathbf{J}-\frac{i}{k_{1} Z_{1}}(\nabla \cdot \mathbf{M}) \mathbf{n} .
\end{gathered}
$$

The extinction, scattering, and absorption cross sections are then derived by integrating the relevant Poynting vectors on the particle surface [37], namely,

$$
\begin{aligned}
& \sigma_{\text {ext }}=-\frac{1}{2} \int_{S} \operatorname{Re}\left[\left(\mathbf{E}_{\text {inc }} \times \mathbf{H}_{\text {surf }}^{*}+\mathbf{E}_{\text {surf }} \times \mathbf{H}_{\text {inc }}^{*}\right) \cdot \mathbf{n}\right] \mathrm{d} S, \\
& \sigma_{\text {sca }}=\frac{1}{2} \int_{S} \operatorname{Re}\left[\left(\left(\mathbf{E}_{\text {surf }}-\mathbf{E}_{\text {inc }}\right) \times\left(\mathbf{H}_{\text {surf }}-\mathbf{H}_{\text {inc }}\right)^{*}\right) \cdot \mathbf{n}\right] \mathrm{d} S, \\
& \sigma_{\text {abs }}=-\frac{1}{2} \int_{S} \operatorname{Re}\left[\left(\mathbf{E}_{\text {surf }} \times \mathbf{H}_{\text {surf }}^{*}\right) \cdot \mathbf{n}\right] \mathrm{d} S,
\end{aligned}
$$

where $\mathbf{E}_{\text {inc }}, \mathbf{H}_{\text {inc }}$ are the incident fields. This provides a fast and accurate way of computing those important properties.

From the surface fields, we can also derive the average field intensity enhancement factor (EF) as $\left\langle\left|\mathbf{E}_{\text {surf }}\right|^{2} /\left|E_{0}\right|^{2}\right\rangle$, where \langle\rangle denotes surface averaging. The parallel [first term in Eqs. (7) and ()] and perpendicular (second term) components may be considered separately.

\section{ACCURACY OF THE MATRIX ELEMENTS}

We first focus on the accuracy of the $\mathbf{D}$ and $\mathbf{K}$ matrix elements used to construct the full SIE interaction matrix. We focus on the integrals involving Green's function of the embedding domain (with relative permittivity chosen as $\epsilon_{1}=1$ ), as they are not strongly dependent on permittivity. For illustration purposes, we will consider a cuboid, shown in Fig. 1(d), as it provides a variety of configurations between pairs of elements. To avoid wave effects, the structure and, thus, the triangular elements are made much smaller than the simulation wavelength. The cuboid dimensions are $20 \mathrm{~nm} \times 5 \mathrm{~nm} \times$ $5 \mathrm{~nm}$, whereas the wavelength is $\lambda=500 \mathrm{~nm}$. The surface of the cuboid is discretized into 160 triangles. To study the accuracy of the reaction integrals, we classify the triangle pairs $\left(T, T^{\prime}\right)$ into the following four groups, which we identified as having different convergence properties:

- Identical triangles, i.e., $T=T^{\prime \prime}$.

- Adjacent triangles, when $T$ and $T^{\prime}$ share a common edge.

- Touching triangles, when $T$ and $T^{\prime}$ share only a common vertex.

- Other triangles in all the other cases.

The cuboid example conveniently contains several pairs in each of these groups, including coplanar, noncoplanar, and orthogonal configurations. The integral accuracy, as estimated from the relative error with respect to the value obtained for the largest quadrature order $\left(n_{O}=19\right)$, is studied as a function of the number of quadrature points $N_{q}$. Note, however, that computing times scale approximately quadratically with $N_{q}$ for double integrals.

Figure 1 shows the results obtained for the integrals pertaining to the $\mathbf{D}$ matrix. It is clear that the Dunavant quadratures significantly outperform uniform quadratures. This should not be surprising, since the Dunavant quadrature is specifically designed to capture higher-order polynomial approximations of the integrand with increasing order of integration. We will therefore only focus on Dunavant quadratures in the rest of this study. Moreover, for all triangle pairs, relative errors of $10 \%$ or better are obtained, even with a single integration point $\left(N_{q}=1\right)$. If a higher accuracy is required, then this is easily achieved with a small number of points for most triangle pairs, but convergence is slower for pairs with a common edge or vertex. Overall, using $N_{q}=61$ (order 17) for these pairs and $N_{q}=7$ (order 5) for the others will guarantee a relative error of $10^{-4}$ or better for the entire $\mathbf{D}$ matrix. In addition, for identical triangles, we compare in Fig. 1(a) the standard SS method with the alternative approach with $4 \mathrm{D}$ analytical evaluation of the singular part of the integral [36]. The latter provides improved precision and is also marginally faster, so it should be preferred.

A similar study can be carried out for the $\mathbf{K}$ matrix (the results of which are shown in Fig. 2). The situation is slightly more complicated here. The integrals are zero for pairs of coplanar triangles [25], which includes identical triangles, so these special cases should not be calculated but enforced to be zero and are therefore excluded from the analysis. In addition, as seen in Fig. 2(a), a small number of integrals exhibit an abnormally large relative error. A closer look indicates that these problematic integrals are in fact much smaller in magnitude than the average integrals and therefore are unlikely to affect the final results. A fairer estimate of the error in this case is by normalizing the absolute error to a typical magnitude of the $\mathbf{K}$ matrix, which we chose as the rootmean-square average of the modulus of the nonzero matrix elements. As seen in Fig. 2(a), this normalized error is of the same order as the relative error in most cases but not affected by the small-magnitude integrals, so it is used in Fig. 2 to characterize the errors in the $\mathbf{K}$ matrix elements.

It is clear from Fig. 2(b) that the integrals for $\mathbf{K}$ can be highly inaccurate in the case of triangles with a common edge. The normalized error can be of the order of $100 \%$ with one 
D matrix
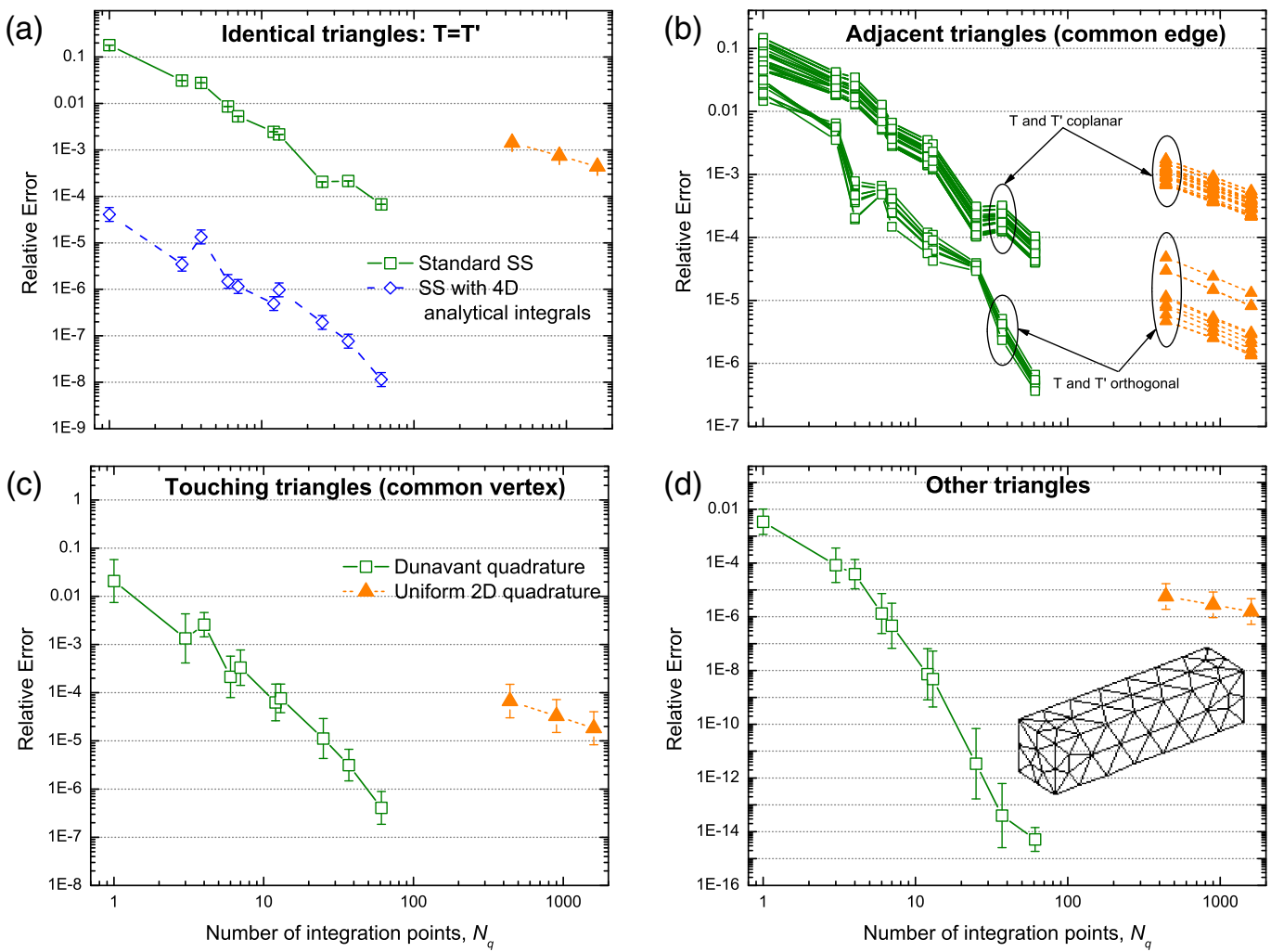

Fig. 1. Variation of the relative errors of the $\mathbf{D}$ matrix integrals as a function of the number of quadrature points per triangle for a cuboid mesh of 160 triangles, as shown in (d). Four cases are distinguished, depending on the pair of source and test triangles: (a) identical triangles; (b) adjacent triangles sharing an edge; (c) touching triangles sharing a vertex; and (d) other triangles. The Dunavant quadrature (open symbols) is compared with the uniform triangular quadrature (filled triangles), which is clearly much worse. In (b), the data are shown explicitly for 48 individual pairs of triangles out of a total of 480 pairs with a common edge to highlight the two different groups corresponding to coplanar and noncoplanar pairs (the latter are located along sharp edges of the rectangle). In (a), (c), and (d) we show the statistical analysis of the log of the error over 160 (a) or 1400 (c),(d) pairs of triangles; i.e., the symbols represent the geometric mean and the error bars the standard deviation of the log of the error.

integration point and remains as large as $1 \%-10 \%$ with $N_{q}=61$. This slow convergence is in fact associated with a remaining logarithmic singularity in the numerical integral, which can be overcome using the SSL method introduced in [25]. Figures 2(b) and 2(c) also show the errors obtained using this method. It is evident that this method improves the accuracy for adjacent triangles by a factor as large as 100 when using $N_{l}=68, N_{q}=61$, which results in errors of $0.01 \%-0.1 \%$ only. Interestingly, as seen in Fig. 2(c), for triangles with only a common vertex (and for all nonadjacent triangles), the line integral formulation does not offer any improvement and is therefore not necessary.

Finally, we note that Figs. 1 and $2 \underline{2}$ can also be obtained for the matrices/integrals corresponding to the second domain, i.e., with the Green's function of the metal (with $\left.\epsilon_{2}=-8.5+0.4 i\right)$. They are not shown here, as they are virtually identical to those obtained for the free-space Green's function. This can be understood because the integration problems are primary geometrical and associated with the $1 / R$ dependence in Green's function, not the $\exp (i k R)$ factor, which is, anyway, almost constant over a given triangle in meshes relevant to plasmonics.

\section{PROPOSED ALGORITHM}

Based on these results, we propose the following algorithm to compute the SIE matrix elements to reasonable accuracy without making the computation too expensive. Two Dunavant quadratures are used, depending on accuracy requirements: $n_{O}=5, N_{q}=7$ for low precision and $n_{O}=17$, $N_{q}=61$ for high precision. The integrals are computed as follows:

- All the double integrals on identical triangles for $\mathbf{D}$ are computed from the full analytical formulas [36] for the singular part of the $4 \mathrm{D}$ integrals and numerically with $n_{O}=17$ for the smooth part. A lowest order would give the same accuracy for small triangles but would not result in significant speed improvements. For $\mathbf{K}$, these integrals are zero.

- All the double integrals on triangles sharing a common edge are computed using the SS method with high-precision $\left(n_{O}=17\right)$ quadrature for the numerical integration over test triangles. Moreover, for $\mathbf{K}$, the SSL line integral formulation proposed in [25] is used, with a 1D Gauss-Legendre quadrature with $N_{l}=4 n_{O}=68$ integration points.

- All the double integrals on triangles sharing a common vertex are computed using the SS method and $n_{O}=17$. The line integral formulation is not needed for these.

- All other double integrals are computed using the SS method and low-precision quadrature $\left(n_{O}=5\right)$.

- Finally, as pointed out in [25], the SS method is not necessary for triangles that are sufficiently far from each other. No singularity subtraction is therefore applied if the following geometric criterion is met: $d>3\left(p_{1}+p_{2}\right) / 6$, where $d$ is the 


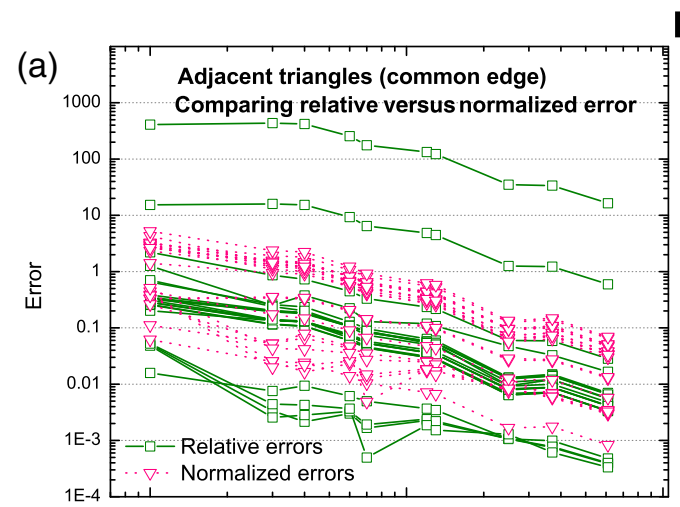

K matrix
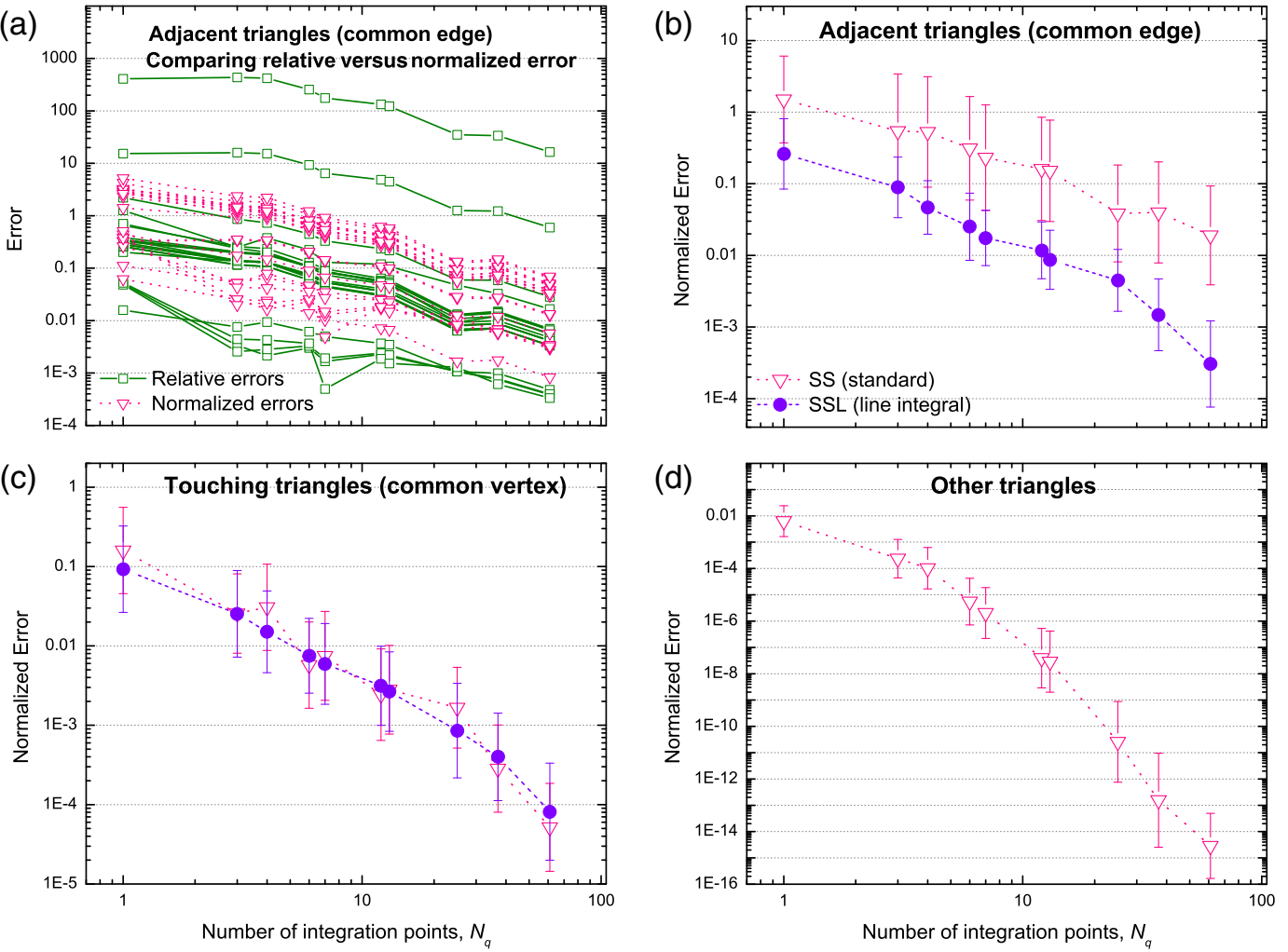

Fig. 2. Variation of the errors in the $\mathbf{K}$ matrix integrals as a function of the number of integration points using Dunavant quadratures for the same cuboid mesh as in Fig. 1. To avoid artificial problems with low-magnitude matrix elements, we compute the normalized error as the absolute error normalized to the root mean square of the $\mathbf{K}$ matrix elements. The two measures are compared in (a) for 21 pairs of adjacent triangles sharing an edge (which are located along sharp edges). The other cases are shown in (b)-(d) as the statistical analysis of the log of the error, as in Fig. 1. In (b) and (c), the results from the standard singularity subtraction (SS) and the alternative SS with line integral (SSL) [25] are compared.

center-to-center distance and $p_{1}$ and $p_{2}$ are the perimeters of $T_{1}$ and $T_{2}$. The threshold of 3 was obtained from similar accuracy tests not shown here.

This approach, which we will denote for short acc-SIE (accurate SIE), guarantees a relative accuracy of $10^{-4}$ or better for the vast majority of integrals of the SIE matrix. It could be argued that such a fine accuracy is not needed in most practical cases, but it may be important for some aspects of the problem (such as absorption) and for some geometries (such as those with sharp edges). One could also adjust these choices to specific needs, but it should be noted that the limiting step for realistic calculations with a large number of degrees of freedom in the case of homogeneous domains is not the matrix calculation but its inversion. This is because only $O\left(N^{2}\right)$ matrix elements have to be computed for a structure discretized into $N$ triangular elements, whereas inverting the matrix requires $O\left(N^{3}\right)$ operations. Compromising on matrix accuracy may therefore not result in worthwhile gain in computing speed for challenging problems with many degrees of freedom. However, the situation could be different in the case of other media where Green's tensor calculation is itself a costly operation.

\section{VALIDATION OF THE PROPOSED IMPLEMENTATION}

We will now compare the performance of this proposed optimized algorithm with the approach previously used for plasmonics calculations (and denoted here std-SIE), where the SSL method is not used and numerical integrations are carried out with a single integration point $\left(N_{q}=1\right)$.

\section{A. Symmetry of Matrix Elements}

One relatively straightforward way to assess the accuracy of the SIE matrix is to check the symmetry properties of the matrix elements. In principle, both $\mathbf{D}$ and $\mathbf{K}$ should be symmetric, since Green's tensor itself is symmetric between source and observation points in the case of homogeneous media. This is a consequence of electromagnetic reciprocity [38]. But, numerically, the symmetry is broken when applying the singularity subtraction method, which involves integrating over one triangle analytically and the other numerically. The error in the symmetry of these matrices can therefore indicate their accuracy. Moreover, any significant breakdown of the symmetry could result in unphysical predictions (for example, negative absorption).

The degree of symmetry in the $\mathbf{D}$ and $\mathbf{K}$ matrix elements is shown in Fig. 3. It is evident that the acc-SIE method shows a much higher degree of symmetry compared to the coarse approach for all types of matrix elements.

\section{B. Optical Properties}

We now move on to study the effects of integration quadrature on the accuracy of physical quantities calculated by SIE. We will study two systems relevant to plasmonics: a silver sphere of $30 \mathrm{~nm}$ radius and a tetrahedron of side $40 \mathrm{~nm}$ both embedded in water. Both systems are illuminated by plane waves incident in the $z$ direction and polarized along $x$. The 

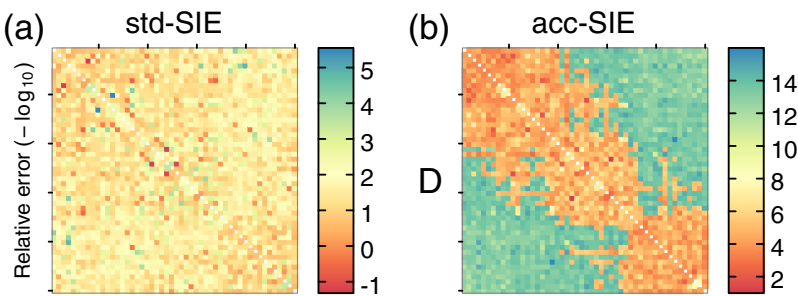

(c)

(d)
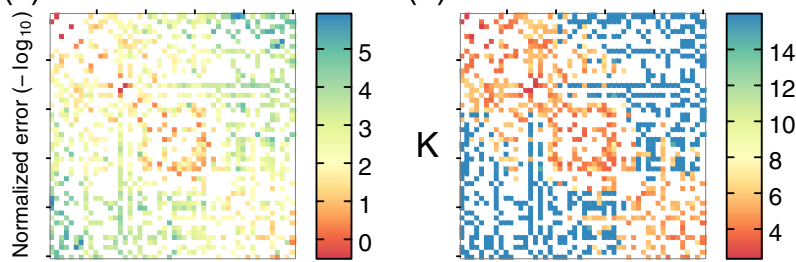

Fig. 3. Symmetry of the matrix elements for the cuboid mesh in Fig. 1(d) obtained from the coarse (std-SIE, $N_{q}=1$, left) and optimized (acc-SIE, $N_{q}=7,61$ with SSL, right) methods presented in this paper. Only a subset of $50 \times 50$ elements are shown for clarity. The color maps represent the number of digits agreement, i.e., $\alpha=$ $-\log 10(\epsilon)$ for a given error $\epsilon$ between transposed elements. The relative error is considered for (a),(b) the D matrix, and (c),(d) the normalized error for the $\mathbf{K}$ matrix. Note the different scales in each panel, and that the error is larger than $100 \%(\alpha<0)$ for some elements in (a), (c).

relative permittivity of water is taken to be constant $(\epsilon=1.77)$ throughout the wavelength range, whereas the relative permittivity of silver is taken from the analytical fit to experimental data $[5,39]$.

The surface of the silver sphere is discretized by meshes of increasing finesse, and the structure is simulated using the proposed optimized algorithm (acc-SIE) and the standard method (std-SIE). The exact optical response of the sphere upon illumination by the plane wave is also calculated using Mie theory [5] and used as a benchmark.

We first consider the wavelength-dependent far- and nearfield properties, which result from the surface plasmon resonances of the nanostructure. Figure $\underline{4}$ summarizes the main results for a representative coarse mesh $(N=240)$ and a finer mesh $(N=840)$. Both the std-SIE and acc-SIE approaches predict the main features of the resonance, even with the relatively coarse mesh. We do, however, observe discrepancies when looking more closely at the details. Notably, the std-SIE approach exhibits significant errors in the quantitative determination of the absorption cross section and of the perpendicular and parallel components of the average local field intensity EF. The acc-SIE approach is much better in this respect, although the finer mesh $(N=848)$ is necessary to reproduce closely the exact results. This can be attributed to the fact that the coarser mesh is not yet an accurate approximation to the spherical geometry.

In order to study the accuracy more quantitatively, we compute the error in the optical properties for various mesh finesses. We focus on a single wavelength, $\lambda=470 \mathrm{~nm}$, which is close to resonance but not at resonance to avoid placing too much emphasis on errors associated with resonance shifts. The convergence properties of both approaches (std-SIE and acc-SIE) are presented in Fig. 5 . It can be seen that the std-SIE approach provides an accuracy almost comparable to the acc-SIE approach for the extinction cross section and the average field intensity on the surface. However, it is an

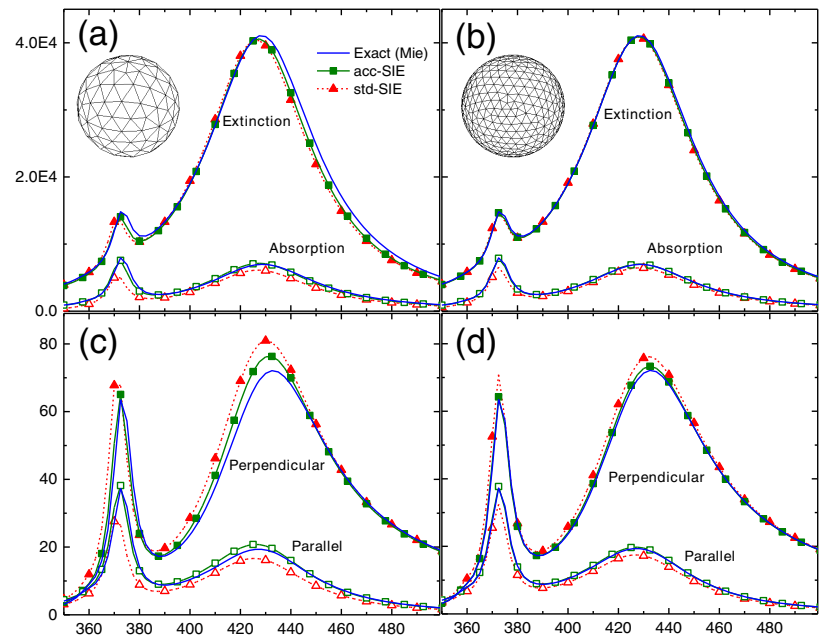

Fig. 4. Wavelength dependence of (a),(b) the far-field and (c), (d) near-field optical properties for a $60 \mathrm{~nm}$ diameter silver sphere in water modeled with SIE utilizing the std-SIE and acc-SIE approaches. Panels (a) and (c) show the results for a coarse mesh $(N=240)$, whereas panels (b) and (d) have a finer mesh $(N=848)$.

order-of-magnitude less accurate in terms of predicting the absorption cross section and the parallel component of the surface field. Even with a mesh refinement of 2400 triangles, the std-SIE method shows an error of $5 \%$ or more, whereas the acc-SIE is better by an order of magnitude. Although comparable accuracy could eventually be reached with the std-SIE approach, it would require a much finer mesh and therefore more CPU/memory resources. We note that the same conclusions would be obtained by doing the study exactly at resonance ( $\lambda=429 \mathrm{~nm}$, not shown here).

In contrast with a sphere, a tetrahedron contains, as with many other objects relevant to plasmonics, sharp corners and edges, around which the fields are expected to vary significantly. One therefore expects numerical predictions to be much more challenging. To capture the field variations reasonably well using linear basis functions, the mesh has to be refined near the edges and corners. The mesh we used to simulate the tetrahedron contains 4680 triangles and is shown in Fig. 6(d). The wavelength dependence of the extinction and absorption cross sections and average surface field intensity as computed by both the std-SIE and acc-SIE approaches are plotted in Fig. 6. Despite the finesse of the mesh, the std-SIE approach clearly fails to predict any physical results. In particular, the absorption cross section is found to be negative. This sort of nonphysical behavior arising from the inaccuracy of SIE using coarse quadratures has been previously reported [40]. In comparison, the acc-SIE approach shows smooth behavior without any such glaring nonphysical results (the accuracy of the results would, nevertheless, need to be checked by considering finer meshes, but this is outside the scope of this work). The particularly poor behavior of the std-SIE approach can be understood from the fact that the optical properties here are likely to be sensitive to potentially large errors in the integration of nearby elements around the edges and corners, which play a significant role in the optical properties.

We note that sharp edges or corners may be an idealization of realistic nanostructures, which often exhibit some degree of rounding of the edges and corners. They are, nevertheless, an important theoretical tool, as rounding of the edges 
(a)
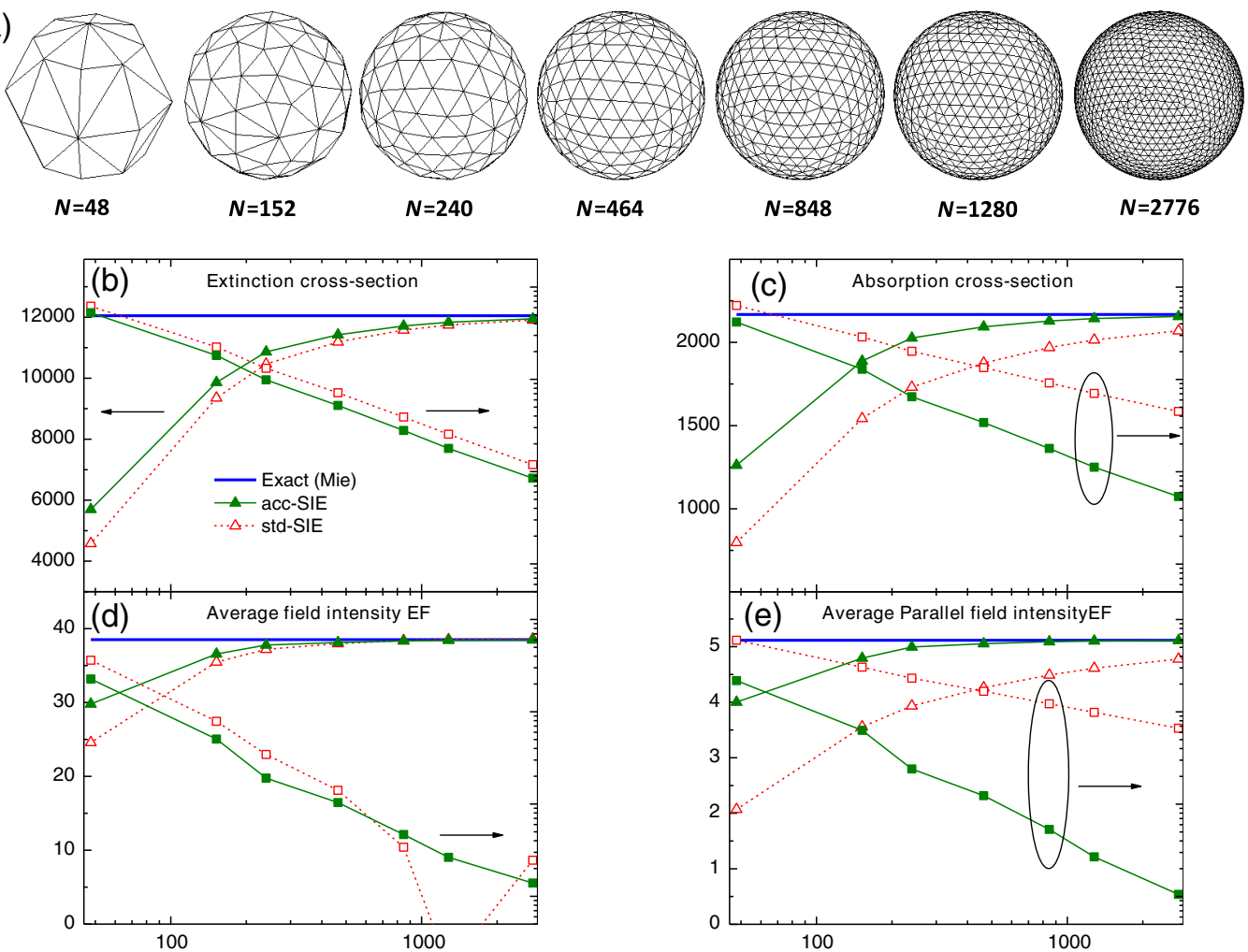

Fig. 5. Comparison between the results calculated by SIE utilizing the std-SIE and acc-SIE approaches for a 60 nm diameter silver sphere in water at $\lambda=470 \mathrm{~nm}$, as a function of the number of triangles on the mesh. (a) Seven meshes of increasing finesse are considered. The properties studied are the (b) extinction and (c) absorption cross sections, (d) the average electric field intensity, and (e) the average parallel electric field intensity. The relative error is obtained by comparison with the exact results of Mie theory.

(a)

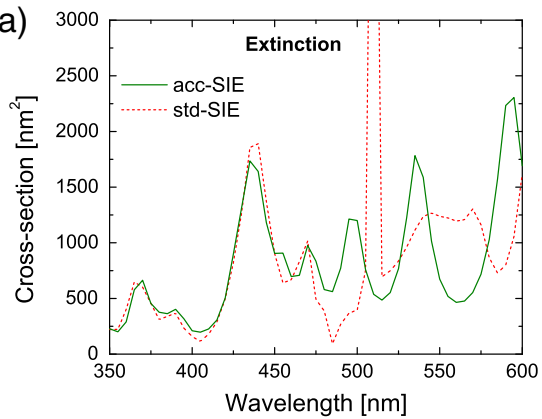

(c)

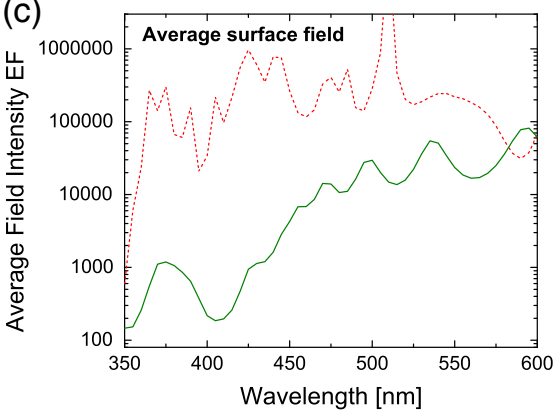

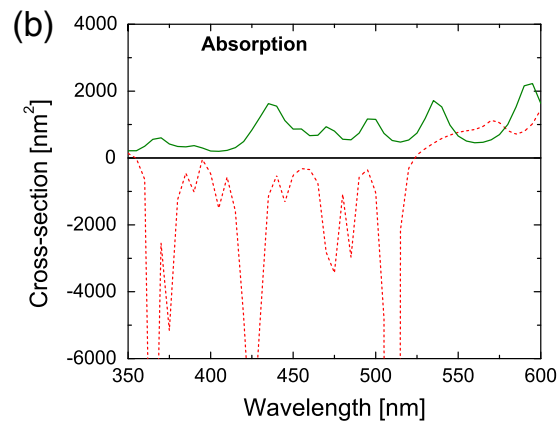

(d)

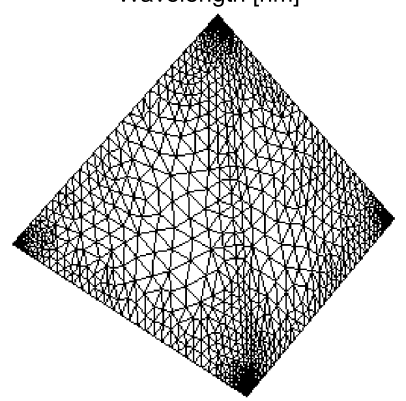

Fig. 6. Wavelength dependence of the optical properties for a silver regular tetrahedron of $40 \mathrm{~nm}$ side embedded in water, computed using the stdSIE and acc-SIE approaches. (a) Extinction cross section. (b) Absorption cross section. (c) Average surface field intensity. (d) Mesh used for the calculation.

introduces additional parameters that are often difficult to measure experimentally. Moreover, the modeling of structures with sharp edges or corners will be necessary to understand the consequences of such rounding on the optical properties.

\section{CONCLUSION}

We have analyzed the effect of the integral quadrature on the accuracy of SIE matrix elements and the physical quantities calculated by SIE. This led us to propose an optimized 
algorithm to evaluate SIE matrix elements to a high accuracy. The optimized algorithm involves some extra computational overhead, but it becomes negligible for the most challenging cases where a large number of degrees of freedom is required. Even though a coarse integration approach might be sufficient for studying the qualitative behavior of some systems, quantitatively it performs poorly in comparison to the improved algorithm. The improved algorithm performs significantly better in calculating properties such as absorption and parallel fields and for geometries with sharp corners and edges, as often considered in plasmonics. It is necessary to use the improved algorithm to obtain any sort of meaningful results in such challenging cases, as shown here with the example of a tetrahedron.

\section{ACKNOWLEDGMENTS}

Funding from the Swiss National Science Foundation (Project 200020_135452) is gratefully acknowledged. ECLR is indebted to the Royal Society of New Zealand (RSNZ) for support through a Marsden Grant and Rutherford Discovery Fellowship.

\section{REFERENCES}

1. S. A. Maier, Plasmonics: Fundamentals and Applications (Springer, 2007).

2. J. Homola, "Surface plasmon resonance sensors for detection of chemical and biological species," Chem. Rev. 108, 462-493 (2008).

3. K. R. Catchpole and A. Polman, "Plasmonic solar cells," Opt. Express 16, 21793-21800 (2008).

4. M. W. Knight, H. Sobhani, P. Nordlander, and N. J. Halas, "Photodetection with active optical antennas," Science 332, 702-704 (2011).

5. E. C. Le Ru and P. G. Etchegoin, Principles of SurfaceEnhanced Raman Spectroscopy and Related Plasmonic Effects (Elsevier, 2009).

6. M. Kauranen and A. V. Zayats, "Nonlinear plasmonics," Nat. Photonics 6, 737-748 (2012).

7. K. Yee, "Numerical solution of initial boundary value problems involving Maxwell's equations in isotropic media," IEEE Trans. Antennas Propag. 14, 302-307 (1966).

8. A. Taflove and M. E. Brodwin, "Numerical solution of steadystate electromagnetic scattering problems using the timedependent Maxwell's equations," IEEE Trans. Microwave Theory Tech. 23, 623-630 (1975).

9. P. Monk, Finite Element Methods for Maxwell's Equations (Oxford University, 2007).

10. W.-H. Yang, G. C. Schatz, and R. P. Van Duyne, "Discrete dipole approximation for calculating extinction and Raman intensities for small particles with arbitrary shapes," J. Chem. Phys. 103, 869-875 (1995).

11. O. J. F. Martin and N. B. Piller, "Electromagnetic scattering in polarizable backgrounds," Phys. Rev. E 58, 3909-3915 (1998).

12. R. Boyack and E. C. Le Ru, "Investigation of particle shape and size effects in SERS using T-matrix calculations," Phys. Chem. Chem. Phys. 11, 7398-7405 (2009).

13. N. G. Khlebtsov, "T-matrix method in plasmonics: an overview," J. Quant. Spectrosc. Radiat. Transfer 123, 184-217 (2013).

14. F. J. García de Abajo and A. Howie, "Retarded field calculation of electron energy loss in inhomogeneous dielectrics," Phys. Rev. B 65, 115418 (2002).

15. U. Hohenester and J. Krenn, "Surface plasmon resonances of single and coupled metallic nanoparticles: a boundary integral method approach," Phys. Rev. B 72, 195429 (2005).

16. A. M. Kern and O. J. F. Martin, "Surface integral formulation for 3D simulations of plasmonic and high permittivity nanostructures," J. Opt. Soc. Am. A 26, 732-740 (2009).

17. J. M. Taboada, J. Rivero, F. Obelleiro, M. G. Araújo, and L. Landesa, "Method-of-moments formulation for the analysis of plasmonic nano-optical antennas," J. Opt. Soc. Am. A 28, 1341-1348 (2011).
18. W. C. Gibson, The Method of Moments in Electromagnetics (Chapman \& Hall/CRC, 2008).

19. G. Mur, "Absorbing boundary conditions for the finite-difference approximation of the time-domain electromagnetic-field equations," IEEE Trans. Electromagn. Compat. 23, 377-382 (1981).

20. J.-P. Berenger, "A perfectly matched layer for the absorption of electromagnetic waves," J. Comput. Phys. 114, 185-200 (1994).

21. B. Gallinet, A. M. Kern, and O. J. F. Martin, "Accurate and versatile modeling of electromagnetic scattering on periodic nanostructures with a surface integral approach," J. Opt. Soc. Am. A 27, 2261-2271 (2010).

22. Y. P. Chen, W. E. I. Sha, W. C. H. Choy, L. Jiang, and W. C. Chew, "Study on spontaneous emission in complex multilayered plasmonic system via surface integral equation approach with layered medium Green's function," Opt. Express 20, 2021020221 (2012).

23. J. Jin, The Finite Element Method in Electromagnetics (Wiley, 2002).

24. R. D. Graglia, "On the numerical integration of the linear shape functions times the 3-D Green's function or its gradient on a plane triangle," IEEE Trans. Antennas Propag. 41, 1448-1455 (1993).

25. P. Yla-Oijala and M. Taskinen, "Calculation of CFIE impedance matrix elements with RWG and $\mathrm{n} \times \mathrm{RWG}$ functions," IEEE Trans. Antennas Propag. 51, 1837-1846 (2003).

26. I. Hänninen, M. Taskinen, and J. Sarvas, "Singularity subtraction integral formulae for surface integral equations with RWG, rooftop and hybrid basis functions," Prog. Electromagn. Res. 63, 243-278 (2006).

27. A. G. Polimeridis and J. R. Mosig, "On the direct evaluation of surface integral equation impedance matrix elements involving point singularities," IEEE Antennas Wireless Propag. Lett. 10, 599-602 (2011).

28. F. Vipiana, D. R. Wilton, and W. A. Johnson, "Advanced numerical schemes for the accurate evaluation of 4-D reaction integrals in the method of moments," IEEE Trans. Antennas Propag. 61, 5559-5566 (2013).

29. C. Forestiere, G. Iadarola, G. Rubinacci, A. Tamburrino, L. Dal Negro, and G. Miano, "Surface integral formulations for the design of plasmonic nanostructures," J. Opt. Soc. Am. A 29, 2314-2327 (2012).

30. M. G. Araújo, J. M. Taboada, D. M. Solís, J. Rivero, L. Landesa, and F. Obelleiro, "Comparison of surface integral equation formulations for electromagnetic analysis of plasmonic nanoscatterers," Opt. Express 20, 9161-9171 (2012).

31. S. Rao, D. Wilton, and A. Glisson, "Electromagnetic scattering by surfaces of arbitrary shape," IEEE Trans. Antennas Propag. 30, 409-418 (1982)

32. A. J. Poggio and E. K. Miller, "Integral equation solutions of three-dimensional scattering problems," in Computer Techniques for Electromagnetics, R. Mittra, ed. (Pergamon, 1973).

33. Y. Chang and R. F. Harrington, "A surface formulation for characteristic modes of material bodies," IEEE Trans. Antennas Propag. 25, 789-795 (1977).

34. T.-K. Wu and L. L. Tsai, "Scattering from arbitrarily-shaped lossy dielectric bodies of revolution," Radio Sci. 12, 709-718 (1977).

35. D. A. Dunavant, "High degree efficient symmetrical Gaussian quadrature rules for the triangle," Int. J. Numer. Methods Eng. 21, 1129-1148 (1985).

36. P. Arcioni, M. Bressan, and L. Perregrini, "On the evaluation of the double surface integrals arising in the application of the boundary integral method to 3-d problems," IEEE Trans. Microwave Theory Tech. 45, 436-439 (1997).

37. C. F. Bohren and D. R. Huffman, Absorption and Scattering of Light by Small Particles (Wiley, 1983).

38. W. Chew, Waves and Fields in Inhomogenous Media (Van Nostrand Reinhold, 1990).

39. P. G. Etchegoin, E. C. Le Ru, and M. Meyer, "An analytic model for the optical properties of gold," J. Chem. Phys. 125, 164705 (2006).

40. A. Kern and O. J. F. Martin, "Pitfalls in the determination of optical cross sections from surface integral equation simulations," IEEE Trans. Antennas Propag. 58, 2158-2161 (2010). 